\title{
Characteristics of the brief but bright discharges that often occur along the trails of positive leaders
}

\author{
Kaneyoshi Takamatsu, Nobuyuki Takagi, and Daohong Wang \\ Department of Electrical and Electric Engineering, Gifu University, 1-1 Yanagido, Gifu, Japan
}

\begin{abstract}
Characteristics of 273 brief but bright (BB) discharges in 14 upward positive leaders and 75 BB discharges in a downward positive leader documented using a high speed video camera have been studied. On the basis of the two-dimensional (2D) shapes obtained from the high-speed video images, the BB discharges can be grouped into five types. Most of the BB discharges appeared only in one frame of our high speed video, indicating that most of them progress very fast. For those slow progressing BB discharges, some of them are identified progressing at two sides and some only at one side, and their speeds are at the order of $10^{6} \mathrm{~m} / \mathrm{s}$, similar to that of a typical negative dart-stepped leader. No apparent differences have been found between the BB discharges in upward lightning and in downward lightning. For 3 of the 14 upward lightning flashes, we have recorded their channel base current and electric field changes. We have detected the simultaneous current and electric field change for only 1 BB discharge among 32 discharges contained in the 3 upward lightning flashes. We argue that the $\mathrm{BB}$ discharges should not be simply called recoil leaders (RLs).
\end{abstract}

Key words: lightning, positive leaders, upward leader, recoil Leader

\section{Introduction}

As shown by Saba et al. (2008) and Warner et al. (2012), many positive leaders occurred with some brief but bright discharges (BB) along their trails. They classified these discharges as recoil leaders (RLs). As described by Mazur (2002), recoil leaders are negative leaders developed on weakly luminous positive leader branches that became cutoff from the main positive leader channel, and then retrace the channel previously ionized by the positive leader. By contrast, many of the BB discharges reported by Warner et al. (2012), exhibited fine structures like check marks among others, and thus it seems to us that most of these BB discharges cannot be simply classified into conventional RLs. Recently, Mazur et al. (2013) have extended recoil leader as bipolar and bidirectional leaders. However, in this paper, the authors did not mention the conventional definition of the recoil leader and the fine structures of the BB discharges reported by Warner et al. (2012) as well. In this study, we first present a statistics on the structure, length and propagation speed of 273 BB discharges in 14 upward positive leaders and $33 \mathrm{BB}$ discharges in a downward positive leader documented using a high-speed camera, and then argue why the BB discharges can not be simply called recoil leaders and should be generalized in the future.

\section{Observation}

Our observation target is the lightning to a windmill or to its lightning protection tower, as well as to a bridge girder located at Uchinada town in the northern coast area of Japan, as shown in Fig 1. A NAC MEMRECAM GX-8 high-speed camera, with its field of view shown in Fig.2, was 
operated at about a distance of $1.4 \mathrm{~km}$ from the windmill. The MEMRECAM GX-8 recorded the lightning at a frame rate of $40 \mathrm{k}$ fps, or $25 \mu \mathrm{s}$ per frame. The resolution was $216 * 288$ pixels (vertical*horizontal) with 12 bit gray scale amplitude resolution. The electric currents of the lightning were measured by Rogowski coils installed at the bases of the windmill and the tower. The output of the Rogowski coils, with a bandwidth from $1 \mathrm{~Hz}$ to $100 \mathrm{kHz}$ was sampled at 500 $\mathrm{kS} / \mathrm{sec}$ for the windmill coil and $200 \mathrm{kS} / \mathrm{s}$ for the tower coil with an amplitude resolution of 16 bit. Electric field changes due to lightning were recorded by slow antenna and a fast antenna. The time constants of the two antennas are $2.2 \mathrm{~ms}, 47 \mu \mathrm{s}$, respectively, and both sampled at 100 $\mathrm{kS} / \mathrm{s}$ with an amplitude resolution of 16 bit.

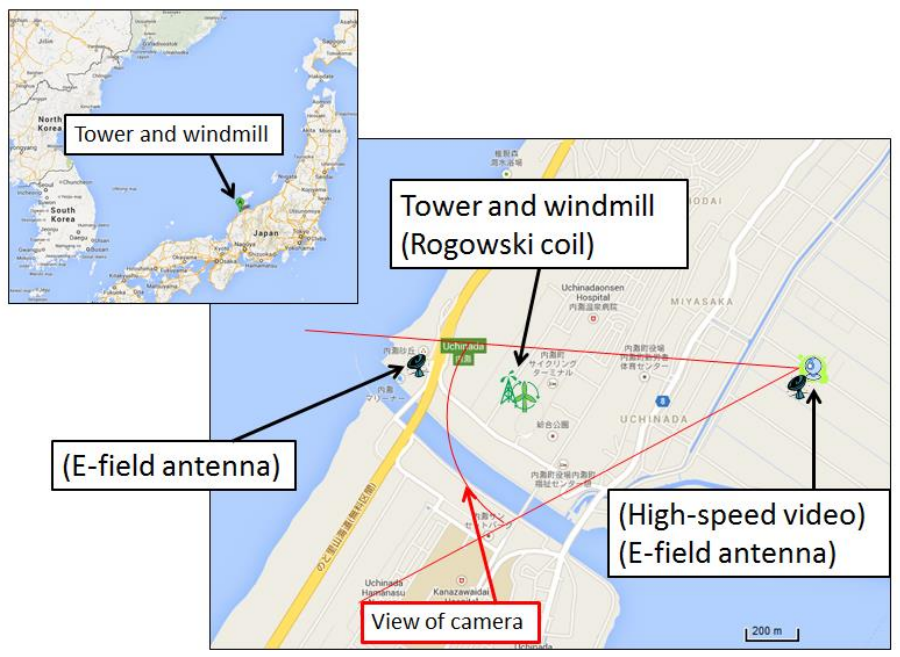

Fig.1 Location of observation.

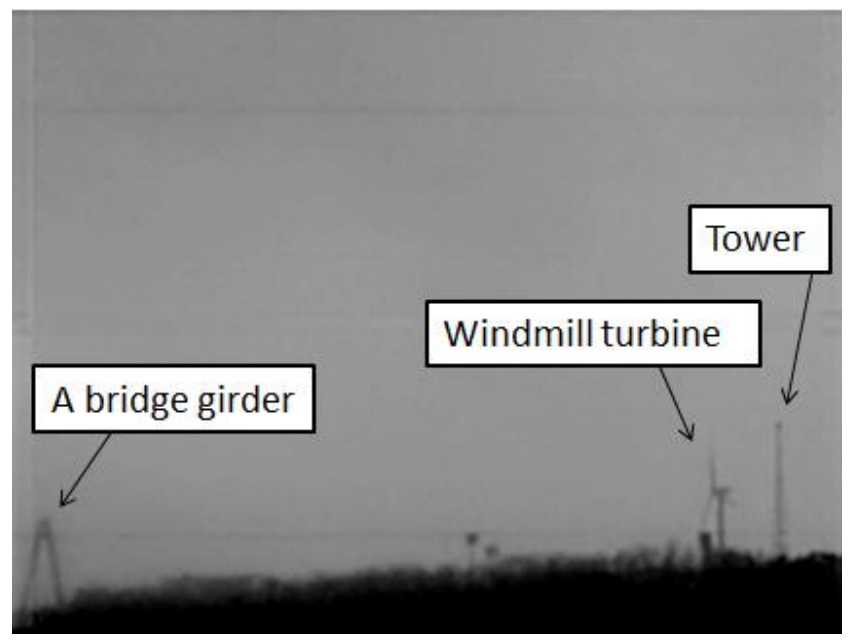

Fig.2 View of high-speed camera. 


\section{RESULTS}

\subsection{General description of our measured data}

Our high-speed camera has been put into operation since November 2011. So far we have recorded 14 upward lightning flashes and 1 downward lightning which exhibited apparent BB discharges along the trails of positive leaders. For 3 of the 14 upward lightning flashes, we have simultaneously recorded their electric currents and electric field (E-field) changes and their BB discharges are listed in Table 1.

Table.1 A list and the classification of the BB discharges contained in 3 upward lightning flashes that have simultaneous recordings of electric currents and E-field changes 2011/12/24 14:51:50 tower and windmil $\quad 1(F)=25(\mu \mathrm{s})$

\begin{tabular}{|c|c|c|c|c|c|c|}
\hline occured time $(+F)$ & shape & duration time $(F)$ & duration time $(\mu \mathrm{s})$ & variation direction & speed $\left(10^{\wedge} 6 \mathrm{~m} / \mathrm{s}\right)$ & length(m) \\
\hline 0 & I & 2 & 50 & - & - & 97 \\
\hline 98 & $\mathrm{~V}$ & 2 & 50 & one side & 2.96 & 104 \\
\hline 161 & I & 1 & 25 & - & - & - \\
\hline 271 & I & 1 & 25 & - & - & - \\
\hline 286 & I & 2 & 50 & one side & 0.17 & 44 \\
\hline 334 & $\mathrm{~V}$ & 4 & 100 & one side & 1.92 & - \\
\hline 363 & I & 1 & 25 & - & - & - \\
\hline 365 & I & 1 & 25 & - & - & - \\
\hline 372 & $\mathrm{~V}$ & 1 & 25 & - & - & - \\
\hline 378 & I & 1 & 25 & - & - & - \\
\hline 440 & $\mathrm{~V}$ & 3 & 75 & one side & 3.52 & 209 \\
\hline 481 & I & 2 & 50 & - & - & - \\
\hline 498 & $\mathrm{~V}$ & 3 & 75 & one side & 0.72 & - \\
\hline 512 & $\mathrm{~V}$ & 2 & 50 & - & - & - \\
\hline 593 & I & 1 & 25 & - & - & - \\
\hline 691 & I & 2 & 50 & - & - & - \\
\hline 1684 & I & 3 & 75 & one side & 1.84 & 114 \\
\hline
\end{tabular}

2012/1/4 $1: 19: 50 \quad$ tower $\quad 1(F)=25(\mu s)$

\begin{tabular}{|c|c|c|c|c|c|c|}
\hline occured time (+F) & shape & duration time $(F)$ & duration time $(\mu \mathrm{s})$ & variation direction & speed $(10 \wedge 6 \mathrm{~m} / \mathrm{s})$ & length $(\mathrm{m})$ \\
\hline \hline 0 & - & 1 & 25 & - & - & 4 \\
\hline 6 & $\mathrm{I}$ & 2 & 50 & - & - & 207 \\
\hline 95 & $\mathrm{~V}$ & 2 & 50 & one side & - & 145 \\
\hline 160 & $\mathrm{I}$ & 2 & 50 & one side & - & - \\
\hline 769 & $\mathrm{I}$ & 1 & 25 & - & - & 44 \\
\hline 1169 & $\mathrm{~V}$ & 3 & 75 & one side & - & 116 \\
\hline 2222 & $\mathrm{I}$ & 6 & 150 & - & - & - \\
\hline
\end{tabular}

$2013 / 1 / 25 \quad 0: 25: 02 \quad$ tower $\quad 1(F)=25(\mu s)$

\begin{tabular}{|r|c|c|c|c|c|c|}
\hline occured time $(+F)$ & shape & duration time $(F)$ & duration time $(\mu \mathrm{s})$ & variation directior & speed $(10 \times 6 \mathrm{~m} / \mathrm{s})$ & length $(\mathrm{m})$ \\
\hline \hline 0 & $\cdot$ & 1 & 25 & - & - & 11 \\
\hline 45 & $\cdot$ & 1 & 25 & - & - & 11 \\
\hline 267 & I & 1 & 25 & - & - & - \\
\hline 577 & Y & 5 & 125 & - & - & - \\
\hline 640 & I & 3 & 75 & one side & 0.96 & - \\
\hline 719 & V & 30 & 750 & one side & - & - \\
\hline 783 & V & 19 & 475 & one side & - & 131 \\
\hline 1423 & I & 4 & 100 & one side & 0.44 & - \\
\hline
\end{tabular}

As an example, Fig. 3 shows the time-integrated image of 14 BB discharges in an upward lightning occurred at $14: 51: 50$ on $12 / 24 / 2011$. The view of the camera is $342 \mathrm{~m}^{*} 432 \mathrm{~m}$ above the tower and the windmill. The lightning was an other-triggered upward lightning simultaneously striking the windmill and the tower (Wang et al, 2008; 2012; Lu et al, 2009). In Fig.4, we labeled the individual BB discharges by solid lines colored in blue to red according to their emergence orders, where purple dashed lines represent main bright channel of the upward lightning. 


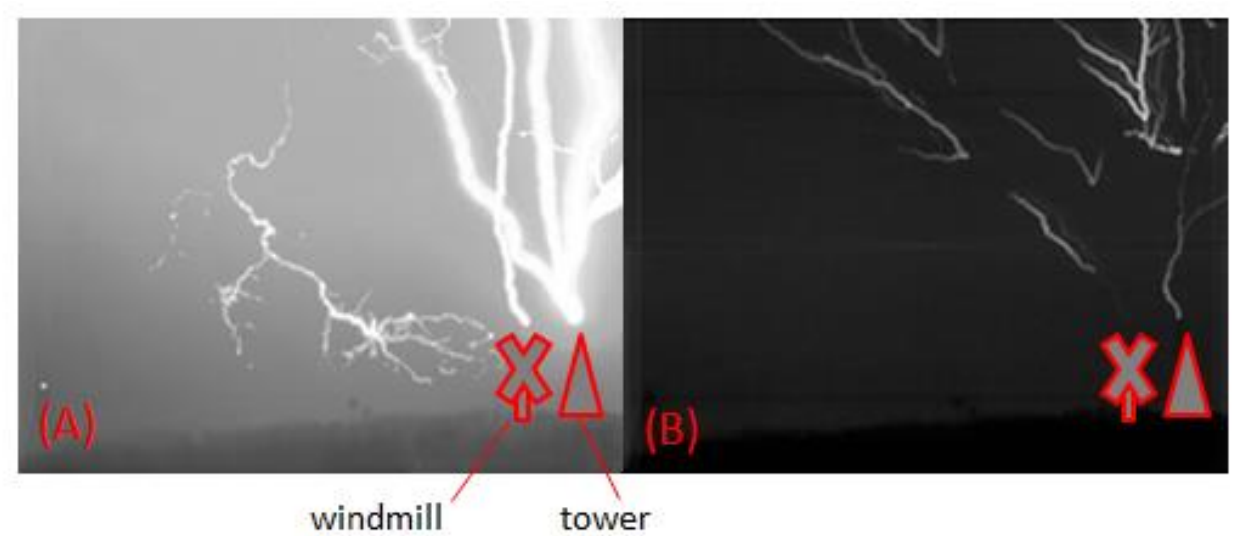

Fig.3 Time-integrated image of lightning occurred on 2011/12/24 (A)main channels (B)BB discharges.

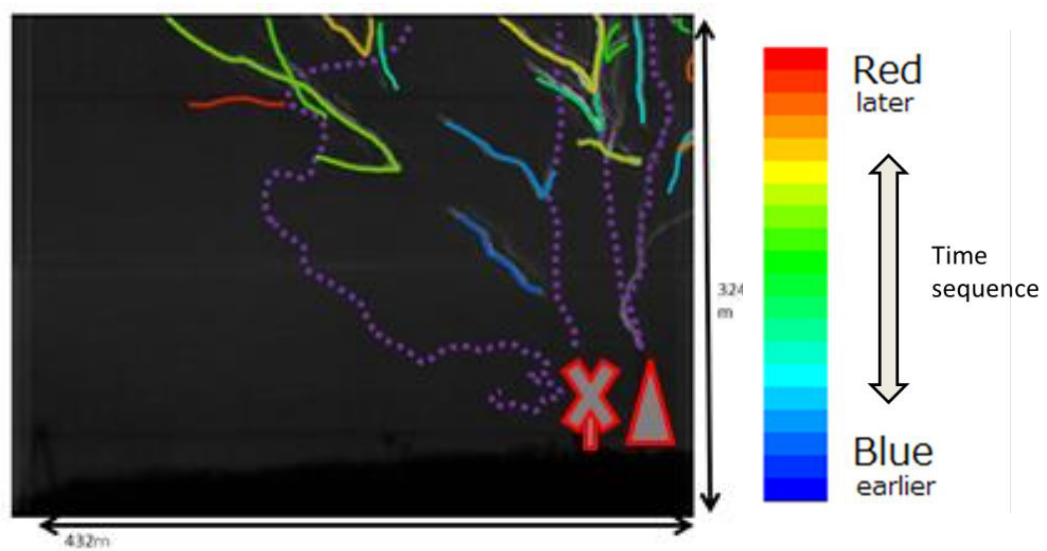

Fig.4 Time-integrated picture of 14 brief discharges occurred in an upward lightning discharge.

Fig.5 gives the time-synchronized current and E-field of the lightning in Fig.3. As clearly shown in Fig.5, this lightning was a combination of an other-triggered upward negative lightning to the tower, and a downward positive lightning to the windmill. The recorded BB discharges shown in Fig. 3 and Fig. 4 occurred during the time interval shadowed in gray starting from 0.016 s on the time scale.

Fig. 6 shows the expanded version of Fig. 5 where gray lines represent the timing of each BB discharge. As evident in this figure, some of BB discharges exhibited detectable electrical current at the base of the lightning channel, whereas others did not. A statistics on this issue will be given in section 3.4 . 

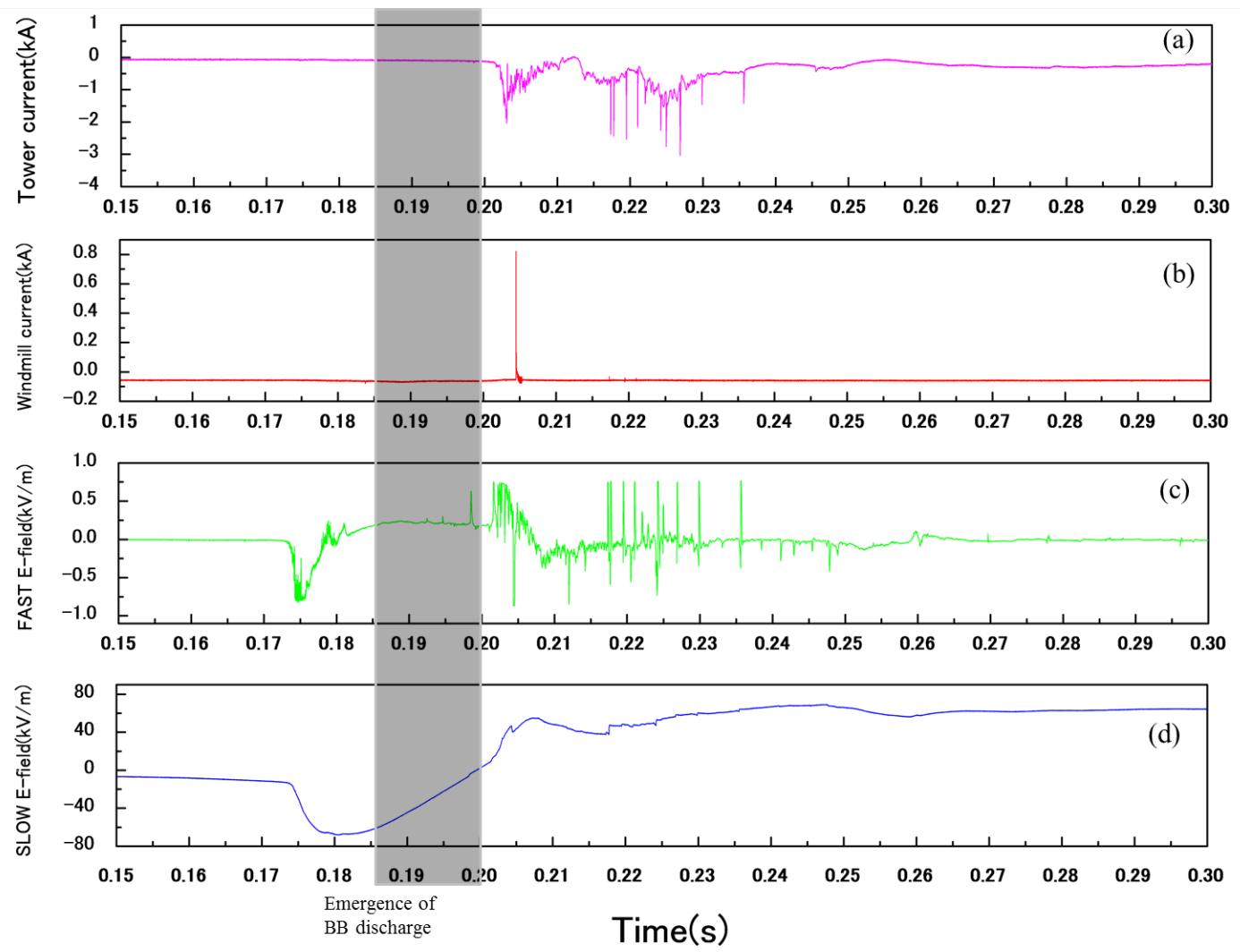

Fig.5 Lightning 2012/12/24 which contained BB discharges with their approximate occurrence times shown in vertical lines. (a)Electric current through the tower (b) Electric current through the windmill (c) E-field change waveforms recorded with fast-antenna (d) E-field change waveforms recorded with slow-antenna. 


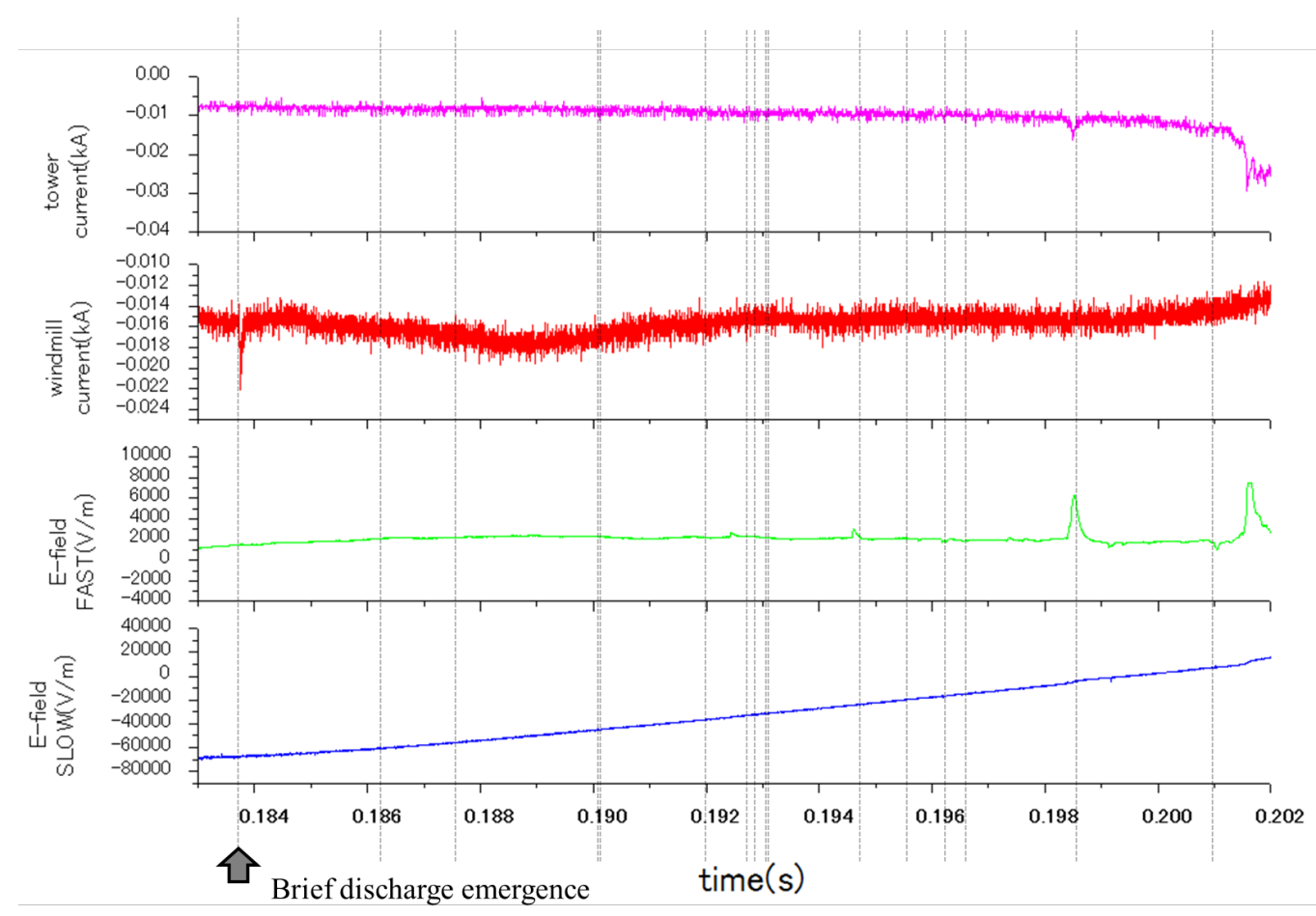

Fig.6 Expansion image of emergence of BB discharge of Fig.5. (a)Electric current through the tower (b) Electric current through the windmill (c) E-field change waveforms recorded with fast-antenna (d) E-field change waveforms recorded with slow-antenna.

As a comparison, Fig.7 shows the time integrated image of BB discharges contained in a downward lightning which occurred at 09:50:12 on 11/19/2012. Before those BB discharges, some continuous downward propagation of positive leaders can be clearly identified.
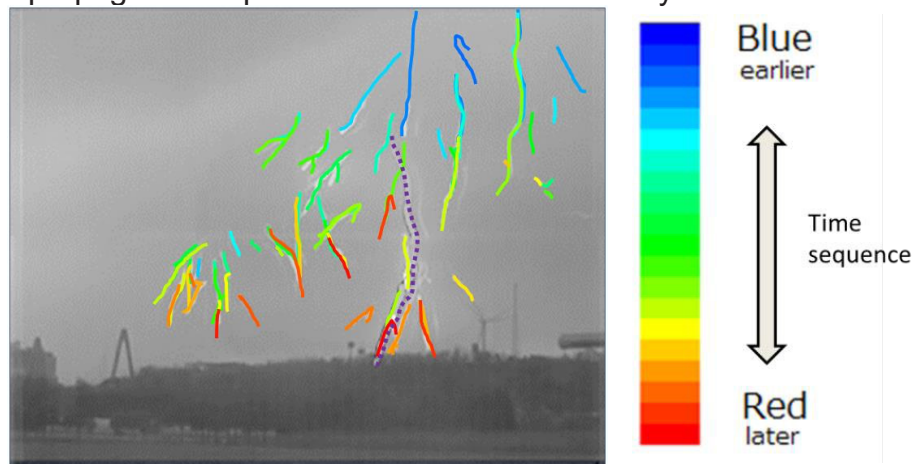

Fig.7 BB discharges in a downward positive lightning discharge. 


\section{2 BB discharge shapes and propagation length}

Fig. 8 shows an example of BB discharge high speed videos and their corresponding light intensity distributions digitized at 8 bits in relative unit. Totally the BB lasted about 4 frames. From Fig.8, we are able to identify the BB integrated shapes and total propagation length. In this study, the BB discharge identification (from background) threshold is 20 digital unit difference in the relative light intensity in Fig.8b.

(a)

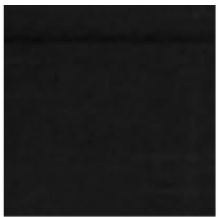

(b)

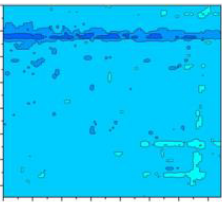

$+0 \mu \mathrm{s}$
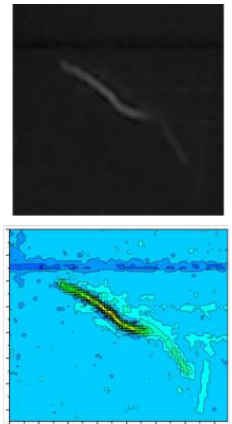

$+25 \mu \mathrm{s}$

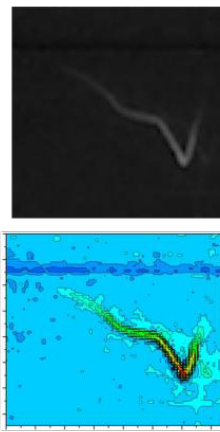

$+50 \mu \mathrm{s}$
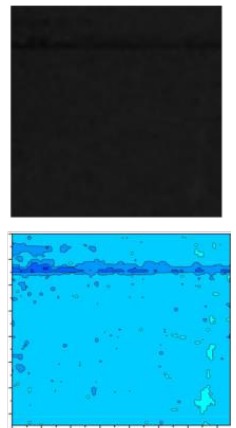

$+75 \mu \mathrm{s}$

Fig.8 Light intensity of BB discharge, (a) Expanded image of each frame of camera, (b) Light intensity of (a).

According to the BB discharge shapes, the BB discharges can be grouped into five types as shown in Fig.9, where they are named V, I, • S, Y, respectively. Fig.10 gives a statistical result on how frequent each shape occurs. As evident from this figure, the majority shapes are I, and there are more $\mathrm{V}$ shapes in upward lightning than in downward lightning.

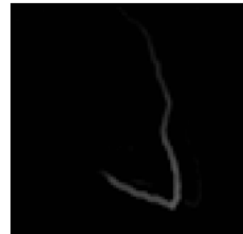

Shape type :V

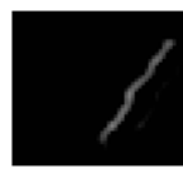

Shape type :I

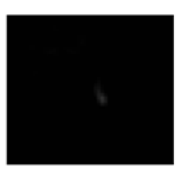

Shape type :

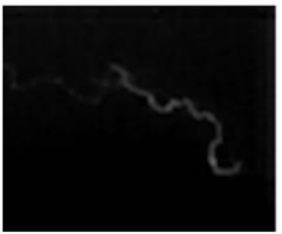

Shape type :S

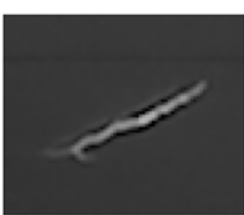

Shape type :Y

Fig. 9 Classification of BB discharge shapes. 

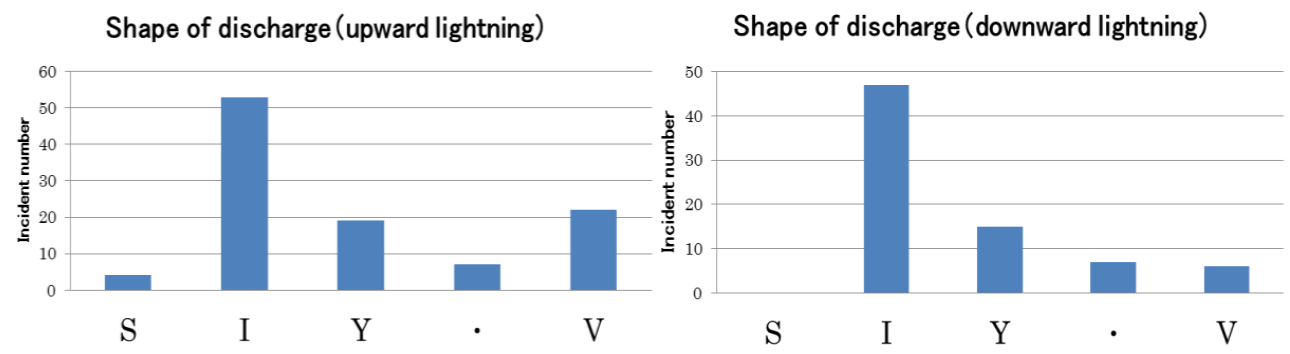

Fig.10 Statistics of BB discharge shapes.

Fig.11 gives histogram of duration time of recorded BB discharge. However, the observation is of two dimensional (2D), we estimated the largest error to be about $30 \%$.
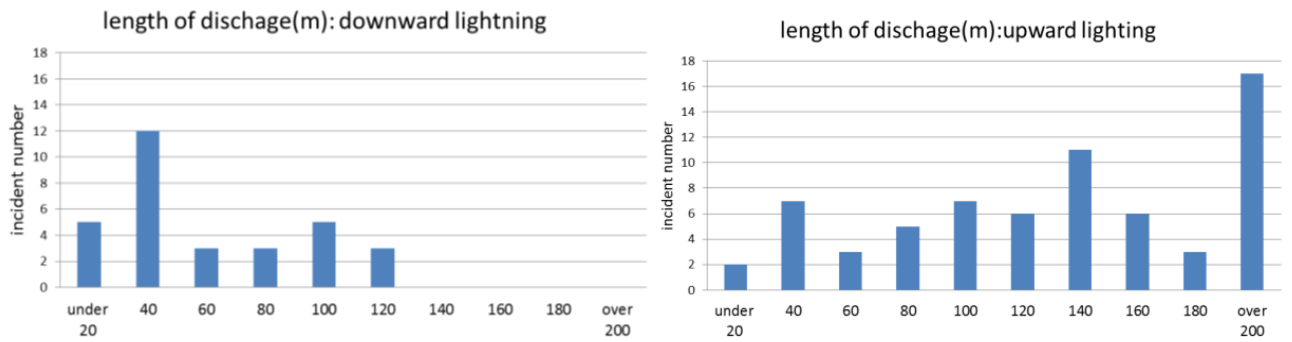

Fig.11 Length of BB discharge (upward/downward).

\subsection{BB discharge propagation direction and speeds}

Also from Fig.8, we are able to identify the propagation direction for 75 BB discharges and the result is shown in Fig.12. 60 of the 75 BB discharges were identified propagating at only one side and the remaining 15 were identified propagating at two sides. For 33 of the 75 BB discharges we are able to further estimate their propagation speeds and the result is shown in Fig.13. The propagation speeds of the BB discharges are found ranging from $0.1^{*} 10^{6}$ to $3^{*} 10^{6}$ $\mathrm{m} / \mathrm{s}$. No difference in the speeds has been found between the upward lightning and the downward lightning. 
Ratio of propagation of all 273 brief discharge

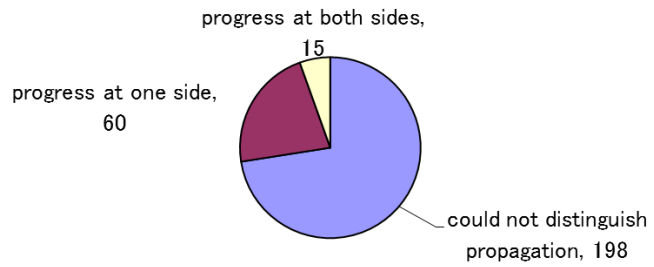

Fig.12 A statistical result on the BB propagation direction.
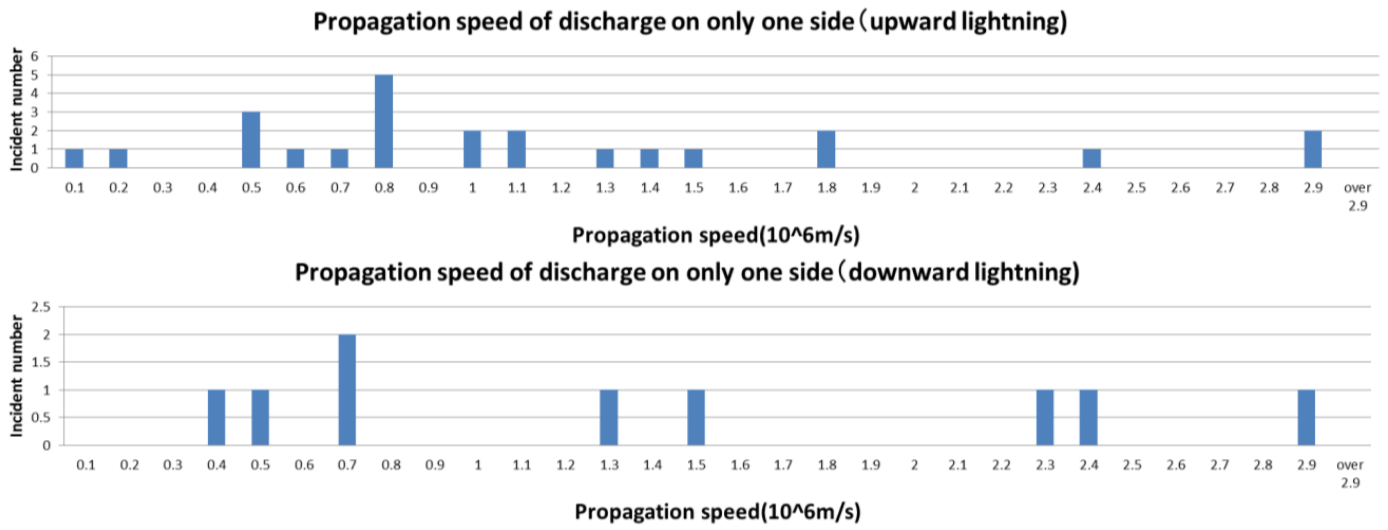

Fig.13 Propagation speeds of BB discharges.

\subsection{Electric current and E-field variation of BB discharge}

Among the 32 BB discharges occurred in three upward lightning for which we have simultaneously recorded the upward lightning current and electric field changes, only 1 of the BB discharges exhibited simultaneously electric current and electric field change detected by our system. For the remaining 31 BB discharges, 2 exhibited only electric current, 7 exhibited only electric field changes and 22 exhibited neither electric current nor electric field changes. For each of the cases, an example waveform is given as shown in Figures 14, 15, 16 and 17. In the case of Fig.14, the electric current reached about 200A and the electric field change reached $400 \mathrm{~V} / \mathrm{m}$. 


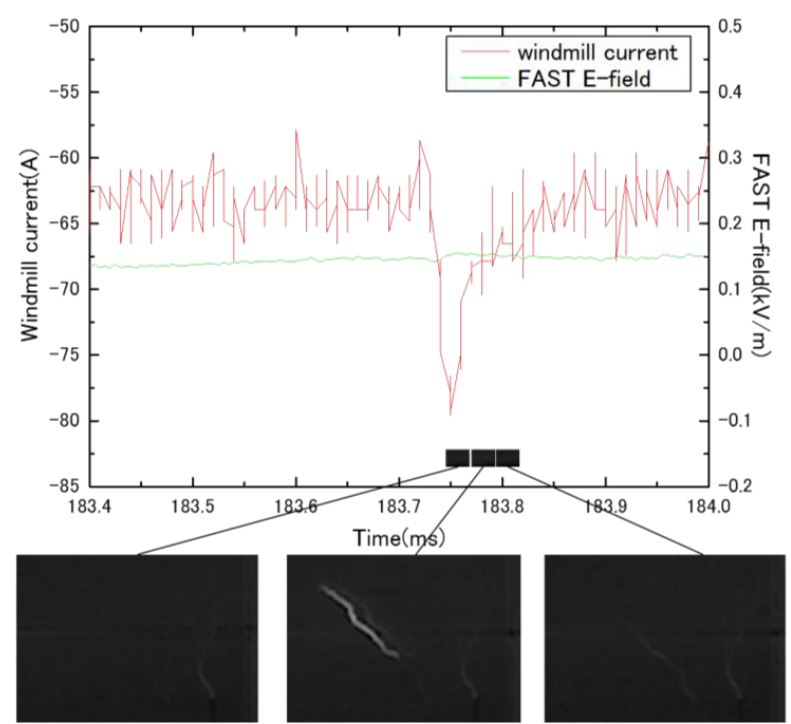

Fig.14 E-field and current of an example BB discharge that exhibited detected current.

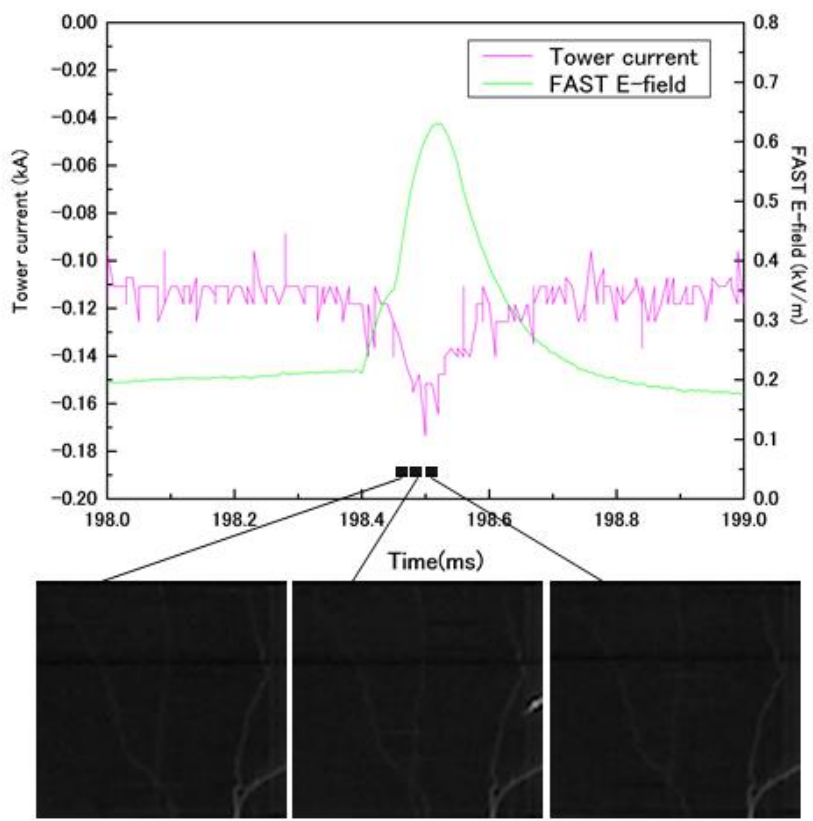

Fig.15 E-field and current of an example BB discharge that exhibited both detected current and E-field change. 


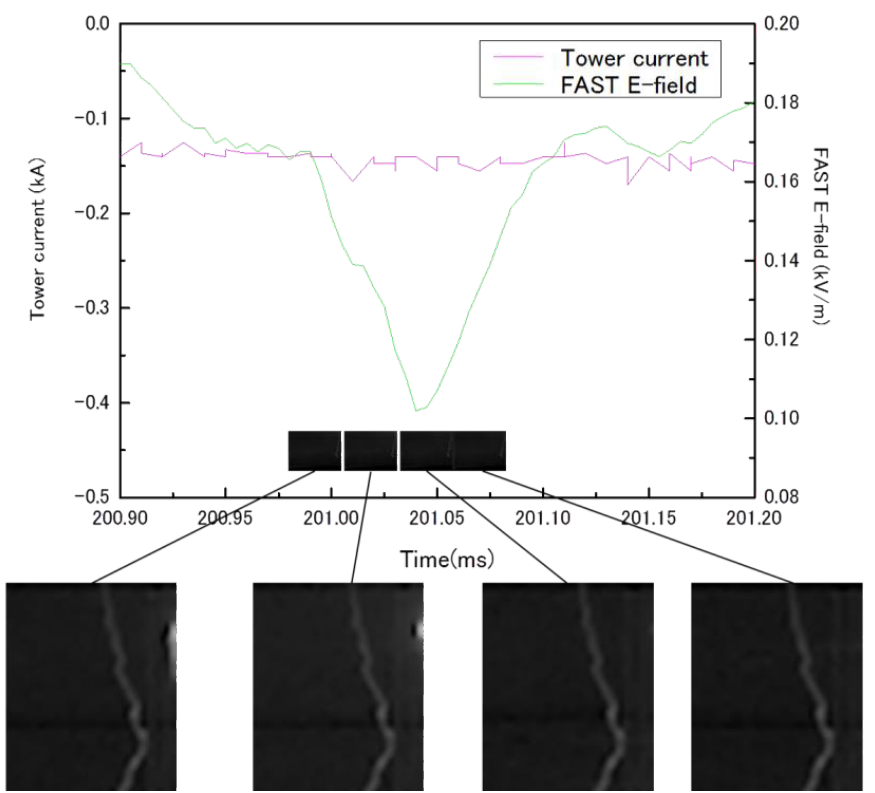

Fig.16 E-field and current of an example BB discharge that exhibited only detected E-field change.

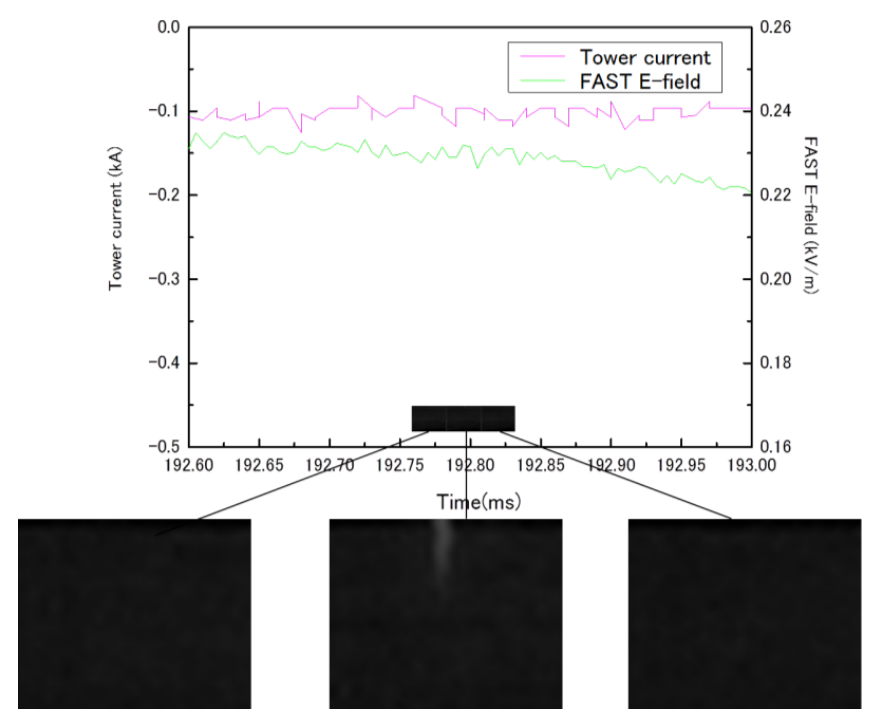

Fig.17 E-field and current of an example BB discharge that exhibited neither detected current nor E-field change. 


\section{Discussion}

Many authors called the BB discharges recoil leaders (Saba et al. 2008). As described by Mazur (2002), recoil leaders are negative leaders developed on weakly luminous positive leader branches that became cutoff from the main positive leader channel, and then retrace the channel previously ionized by the positive leader. Indeed, positive leaders are usually very weak and easily become cutoff from the main positive leader channel. Following the channel cutoff, as suggested by Mazur and Ruhnke (1993), the channel of the positive leader continues its extension at the upper tip while cooling and losing conductivity at the lower tip (in the case of upward lightning), and still maintains its residual net positive charge. Becoming a floating conductor in an ambient electric field, the leader obtains induced charges that distribute as a dipole in addition to the existing residual positive charge. The growth of dipole charges results in a growing negative charge along the decaying lower end of the channel. The process will lead some leader discharges, maybe at two ends of the floating channel as suggested by Mazur el al. (2013). Those leaders sometimes may be bright enough to be recorded and called recoil leaders. However, we should note some of the leaders will attach to the main positive leader channel without much brightness and then induce a return stroke type of bright discharge. Many BB discharges appeared in only one frame of our high speed video and they should have a speed faster than a leader. Thus we think many of these BB discharges should be return-stroke type of discharges, rather than leader type of discharges. Moreover, we should note that two floating channels could simultaneously exist as an example sketched in Figure 18. Due to a possible horizontal electric field, the rejuvenated leaders at the lower ends of the two branches will have opposite polarity and their connection will apparently result in a "V" or even "Y" shape of minireturn stroke. We speculate that the "V" shape of BB discharge shown in Fig.8 is actually such type of mini-return stroke. Based on all these reasons, we think the BB discharges should not be simply called recoil leaders.
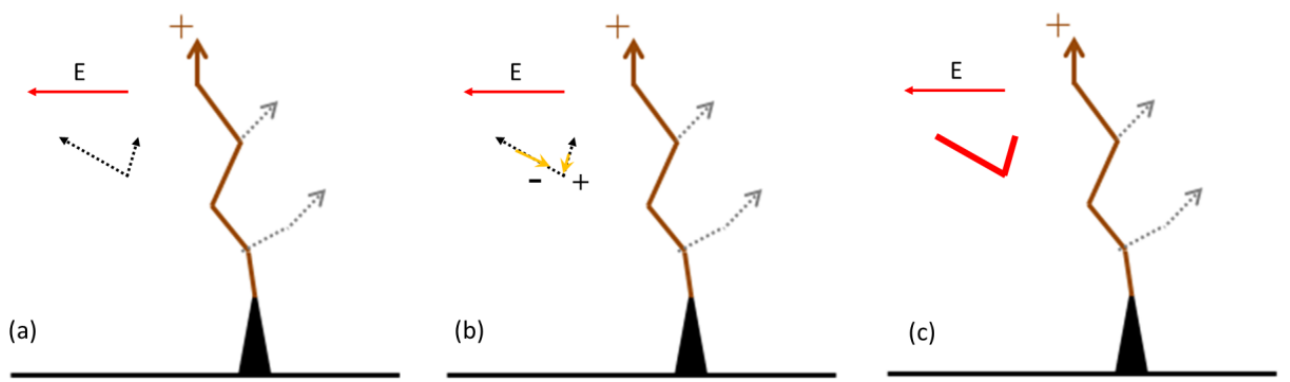

Fig.18 A proposed scenario for formation of $\mathrm{V}$ shape BB discharge. (a) two floated branches; (b) development of leaders at the two branches due to horizontal component of electric field; (c) connection of the two leaders and then a mini-return stroke (BB discharge).

Recent development of lightning mapping technologies has made the mapping of positive leaders possible (Akita et al.2014; Stock et al. 2014). However we should note that in the case of a positive leader which propagates in continuous mode, what were mapped may be just the locations of the BB discharges. In many cases we observed, we can see both the weak continuous propagation of the positive leader itself and the following BB discharges. If we locate all the BB discharges as shown in Fig.7, we can also get a kind of propagation with scattered 
sources. But this propagation is apparently not the preceding continuous propagation of the positive leader itself although they may have similar propagation speeds.

\section{Conclusion}

BB discharges can be grouped into five types according to their 2D bright shapes on high speed videos. Most of the BB discharges appeared only in one frame of our high speed video, indicating that most of them progress very fast. For those slow progressing BB discharges, some of them are identified progressing at two sides and some only at one side (either direction), and their speeds are at the order of $10^{6} \mathrm{~m} / \mathrm{s}$, similar to that of a typical negative dart-stepped leader. No apparent differences have been found between the BB discharges in upward lightning and in downward lightning. In upward lightning, only a few percentage of BB discharges exhibit simultaneous channel bottom current and the related electric field change. We argue that the BB discharges should not be simply called recoil leaders (RLs)

\section{Acknowledgment}

This work was supported by the Ministry of Education, Culture, Sports, Science, and Technology of Japan (Grant2336012) and partly supported by Power Academy Special Research 2013. Authors also thank Dr. Mi Zhou from Wuhan University for his help in improving the manuscript.

\section{Reference}

Akita, M., Michael Stock, Z. Kawasaki, P. Krehbiel, W. Rison, M Stanley; "Data processing procedure using distribution of slopes of phase differences for broadband VHF interferometer"; Journal of Geophysical Research: Atmospheres; Volume 119, Issue 10, pages 6085-6104, 27 May 2014.

Stock, M. G., M. Akita, P. R. Krehbiel, W. Rison, H. E. Edens, Z. Kawasaki,M. A. Stanley; "Continuous broadband digital interferometry of lightning using a generalized crosscorrelation algorithm"; Journal of Geophysical Research: Atmospheres; Volume 119, Issue 6, pages 3134-3165, 27 March 2014.

Saba, M. M. F., Kenneth L. Cummins, T. A. Warner, E. Philip Krider,Leandro Z. S. Campos, Mauricio G. Ballarotti, Osmar Pinto Jr., Stacy A. Fleenor; "Positive leader characteristics from high-speed video observations”; GEOPHYSICAL RESEARCH LETTERS, VOL. 35, L07802, doi:10.1029/2007GL033000, 2008.

Warner, T. A., Marcelo M. F. Saba, and Richard E. Orville; "Characteristics of Upward Leaders from Tall Towers"; 22nd International Lightning Detection Conference.

Mazur, V., L. H. Ruhnke; "Common physical processes in natural and artificially triggered lightning”; J. Geophys. Res. 98 (1993) 12,913-12,930.

Mazur, V.; "Physical processes during development of lightning flashes"; C. R. Physique 3 (2002) 1393-1409.

Mazur, V., Lothar H. Ruhnke, T. A. Warner, Richard E. Orville; "Recoil leader formation and development"; Journal of Electrostatics 71 (2013) 763-768.

Lu, W., D.Wang, Y. Zhang and N. Takagi; "Two associated upward lightning flashes that produced opposite polarity electric field changes”; Geophys. Res. Lett., Vol.36, L05801, doi:10.1029/2008GL036598, 2009. 
Wang, D., N. Takagi, T. Watanabe, H. Sakurano, M. Hashimoto; "Observed characteristics of upward leaders that are initiated from a windmill and its lightning protection tower"; Geophys. Res. Lett. Vol.35, L02803, doi:10.1029/2007GL032136, 2008.

Wang, D., N. Takagi; "Characteristics of winter lighting that occurred on a windmill and its lightning protection tower in Japan, IEEJ Trans. on Power and Energy"; Vol. 132, No,6, pp.568-572, Doi:10.1541/ieejpes.132.568, 2012.

(Received September 15, 2014; revised December 24, 2014; accepted January 13, 2015) 